175 INHIBITON OF TUMOUR NECROSIS FACTOR ALPHA SIGNALLING IMPROVES VASCULAR REMODELLING AND DECREASES THE PRO-INFLAMMATORY AND CYTOTOXIC PHENOTYPE OF PERIPHERAL NATURAL KILLER CELLS IN A MODEL OF CHRONIC HYPERTENSION IN PREGNANCY

Heather Small*, Ryszard Nosalski, Hannah Morgan, Tomasz Guzik, Delyth Graham, Christian Delles. University of Glasgow; *Presenting Author

\subsection{6/heartjnl-2016-309890.175}

Objective Pregnancy induces extensive yet relatively rapid remodelling of the cardiovascular (CV) system, however, little is known about this adaptation in women with pre-existing $\mathrm{CV}$ disease. We have previously characterised the stroke prone spontaneously hypertensive rat (SHRSP) as a model of deficient uterine artery remodelling and identified an increase in pro-inflammatory TNFÎ \pm relative to the normotensive WKY strain during pregnancy.

Design and method SHRSP were treated with etanercept $(0.8$ $\mathrm{mg} / \mathrm{kg}$ ) or vehicle at gestational day (GD) 0, 6, 12 and 18 . Animals were sacrificed at GD18. SHRSP, SHRSP treated with etanercept (ETN) and WKY $(n=6)$ were used for vascular studies. An independent set of animals $(n=6)$ were used for flow cytometry analysis.

Results Etanercept significantly reduced systolic blood pressure in the SHRSP after GD 12 (ÎI"SBP GD 10-21 SHRSP $12.0 \pm$ 4.17 vs. ETN $25.8 \pm 4.27 \mathrm{mmHg}$; $\mathrm{p}<0.05)$. Analysis of GD18 uterine arteries showed that etanercept significantly reduced uterine artery contractile ability (SHRSP $57.3 \pm 8.75$ vs. ETN $35.2 \pm 2.19 \mathrm{kPa} ; \mathrm{p}<0.01)$ and increased carbachol response (SHRSP $13.8 \pm 3.8 \%$ vs. ETN $40.1 \pm 3.25 \%$; p < $0.05)$. Characterisation of uteroplacental blood flow using Doppler showed that etanercept significantly reduced resistance index relative to SHRSP (SHRSP $0.79 \pm 0.02$ vs. ETN 0.61 \pm 0.02 resistance index; $p<0.01)$. Etanercept significantly increased litter size in the SHRSP (SHRSP $7.80 \pm 0.44$ vs. ETN $12.75 \pm 0.94$ fetuses), reduced resorption frequency (SHRSP $66.7 \%$ vs. ETN $25.0 \%$ dams with resorption) and decreased premature glycogen cell loss from the placenta. Further, we sought to identify the source of excess TNFÎ \pm in the SHRSP. Inflammatory natural killer (NK) cells (CD3$\mathrm{CD} 161^{+}$) were significantly increased in the SHRSP relative to the WKY in the placenta (WKY $11.6 \pm 2.39$ vs. SHRSP $659.8 \pm 201.2$ cells $/ \mathrm{mg} ; \mathrm{p}<0.01)$. Etanercept reduced the percentage of NK cells which produced TNFî \pm in the maternal circulation and placenta in the SHRSP. Additionally, etanercept significantly reduced the number of CD $161^{+} \mathrm{NK}$ cells in the placenta of the SHRSP (SHRSP $659.8 \pm 201.2$ vs. ETN $148.0 \pm 12.62$ cells/mg; $\mathrm{p}<0.01$ ) by inducing a phenotypic switch to a granzyme $\mathrm{B}_{\text {low }} \mathrm{CD} 161_{\text {low }}$ population.

Conclusions Etanercept improves uterine artery function and pregnancy outcome in the SHRSP. We propose that this is through the limitation of both damaging TNFI \pm release and cytotoxicity from NK cells.

\section{A HISTONE DEACETYLASE 7-DERIVED 7 AMINO ACID PEPTIDE ACTS AS A PHOSPHORYLATION CARRIER}

Junyao Yang*, Ka Hou Lao, Lingfang Zeng. King's College; *Presenting Author

10.1136/heartjnl-2016-309890.176
Histone deacetylase 7 (HDAC7) belongs to class II HDAC family, playing a pivotal role in the maintenance of endothelium integrity. There are 8 splicing variants in mouse HDAC7 mRNAs. Within the $5 \hat{a} €{ }^{\mathrm{rm}}$ terminal non-coding area of some variants, there exist some short open reading frames (sORFs). Whether these sORFs can be translated and their potential roles in cellular physiology remain unclear. In this study, we demonstrated that one sORF encoding a 7 amino acids (aa)-peptide could be translated in vascular progenitor cells (VPCs) in response to vascular endothelial cell growth factor (VEGF). The 7aa-peptide (7A) could be phosphorylated at serine residue via MEKK1. Importantly, the phosphorylated 7A (7Ap) could transfer the phosphorylation group to the Threonine residue of the $14-3-3 \hat{\mathrm{I}}^{3}$ protein in a cell free in-gel buffer system. The in vitro functional analyses revealed that $7 \mathrm{~A}$ enhanced VEGF-induced VPC migration and differentiation toward endothelial cell (EC) lineage, in which MEKK1 and 14-3$3 \hat{\mathrm{I}}^{3}$ served as the upstream kinase and the downstream effector respectively. Knockdown of either MEKK1 or $14-3-3 \hat{\mathrm{I}}^{3}$ attenuated VEGF-induced VPC migration and differentiation. Exogenous 7Ap could rescue the effect of VEGF on the MEKK1 siRNAtransfected but not on the $14-3-3 \hat{I}^{3}$ siRNA-transfected VPCs. The in vivo studies showed that $7 \mathrm{~A}$ especially $7 \mathrm{Ap}$ induced capillary vessel formation within Matrigel plug assays, increased re-endothelialization and suppressed neointima formation in the femoral artery injury model, and promoted the foot blood perfusion recovery in the hindlimb ischemia model via increasing $\mathrm{Sca} 1^{+}$cell niche formation. These results indicate that the sORFs within the non-coding area can be translated under some circumstances and that the 7aa-peptide may play an important role in cellular processes like migration and differentiation via acting as a phosphorylation carrier.

\section{ENDOTHELIAL MICROPARTICLES: NOVEL REGULATORS OF VASCULAR CALCIFICATION IN VITRO}

${ }^{1}$ Daniel Moreno Martinez*, ${ }^{1}$ Fiona Wilkinson, ${ }^{2}$ Eoghan McCarthy, ${ }^{1}$ Ayman Mahmoud, ${ }^{2}$ Benjamin Parker, 'Yvonne Alexander. 'Cardiovascular Research Group, Healthcare Science Research Centre, Faculty of Science and Engineering, Manchester Metropolitan University \& Manchester Academic Health Science Centre, UK; ${ }^{2}$ Centre for Musculoskeletal Research, University of Manchester, Manchester, UK; *Presenting Author

\subsection{6/heartjnl-2016-309890.177}

Endothelial microparticles (EMPs) are complex structures with pleiotropic properties and are emerging as an index of endothelial damage; however, further work to determine the effect of EMPs on vascular smooth muscle cells (VSMC) is needed. We have shown that elevated EMPs are detected in Systemic Lupus Erythematosus (SLE) and carotid artery disease patients, who present accelerated vascular ageing and calcification. This study aims to investigate the molecular components of EMPs and whether they modulate vascular calcification and osteogenic differentiation of VSMCs in vitro.

EMPs were generated in vitro using human aortic endothelial cells (AoEMPs) by serum starvation $(24 \mathrm{~h})$ followed by TNF-alpha stimulation $(10 \mathrm{ng} / \mathrm{ml} ; 24 \mathrm{~h})$, isolated by ultracentrifugation and quantified using flow cytometry. Human coronary artery smooth muscle cells (HCASMCs) were incubated with $10^{6}$ AoEMPs $/ \mathrm{ml}$ in osteoinductive media (5 $\mathrm{mM} \mathrm{BGP}$ and $2.6 \mathrm{mM} \mathrm{CaCl}_{2}$ ) for 21 days. Calcification was assessed by alizarin red staining and calcium deposition assays. Conditioned media was collected at 7, 14 and 21 days to identify markers of bone metabolism using Bioplex array 